\title{
Optimization of Chemical Bath Deposited Mercury Chromium Sulphide Thin Films on Glass Substrate
}

\author{
H. B. Patil ${ }^{1}$ and S. V. Borse ${ }^{2}$ \\ ${ }^{1}$ Department of Physics, R.C. Patel ASC College, Shirpur 425405, India \\ ${ }^{2}$ Department of Physics, S.S.V.P.s College, Shindkheda, India
}

Correspondence should be addressed to H. B. Patil; h.patil7@gmail.com

Received 28 June 2013; Accepted 11 August 2013

Academic Editors: A. Chatterji and R. S. Mane

Copyright (C) 2013 H. B. Patil and S. V. Borse. This is an open access article distributed under the Creative Commons Attribution License, which permits unrestricted use, distribution, and reproduction in any medium, provided the original work is properly cited.

Semiconducting thin films of ternary $\mathrm{Hg}_{x} \mathrm{Cr}_{2-x} \mathrm{~S}_{4}(x=0.4)$ have been deposited on glass substrate by the simple and economical chemical bath deposition method. We report the deposition and optimization of the solution growth parameters such as temperature, complexing agent, thiourea, and deposition time that maximizes the thickness of the deposited thin film. The Xray diffraction deposited $\mathrm{Hg}_{x} \mathrm{Cr}_{2-x} \mathrm{~S}_{4}$ thin films having cubic structure. The thin films were uniform and adherent to substrate. The composition was found homogeneous and stoichiometric by EDAX analysis.

\section{Introduction}

Mercury chromium sulfide $\left(\mathrm{HgCr}_{2} \mathrm{~S}_{4}\right)$ is a chalcogenide metal sulfide semiconductor of the II-VI group compound semiconductors. The technological interests in polycrystalline-based devices are mainly caused by their low production cost [1]. The use of $\mathrm{HgCr}_{2} \mathrm{~S}_{4}$ thin films of semiconductor has attracted much interest because of their role in variety of applications in various magneto-optical and optoelectronics devices [2] as well as magnetocapacitive or magnetoelectric effect devices [3-7]. Many techniques have been reported in the deposition of thin films such as evaporation, sputtering, spray pyrolysis, molecular beam epitaxy, and photochemical deposition. There is a problem in each of these deposition methods $[8,9]$. Amongst all, chemical bath deposition (CBD) is simple and of low cost and is suitable for a large area deposition [10]. Thin films of diluted magnetic semiconductors attract many researchers due to their wide range of applications in various fields. The films of $\mathrm{HgCr}_{2} \mathrm{~S}_{4}$ are usually crystallized in cubic structure with lattice constants 10.2 $\AA$ [11].

In the present study, the chemical bath process is performed by slow release of $\mathrm{S}^{2-}$ ions and controlled free $\mathrm{Hg}^{2+}$ and $\mathrm{Cr}^{2+}$ ions that react to form $\mathrm{HgCr}_{2} \mathrm{~S}_{4}$ nuclei on glass substrate and in the bath solution in the form of precipitation.
The properties of the deposited thin films basically depend on the deposition parameters such as deposition temperature, complexing agent, thiourea, deposition time, $\mathrm{pH}$ value, composition of materials, and film thickness. Finally, we report the deposition of $\mathrm{HgCr}_{2} \mathrm{~S}_{4}$ thin films and the investigation of the different deposition parameters to obtain uniform film having expected thickness.

\section{Materials and Methods}

In the present investigation, thin films of $\mathrm{Hg}_{x} \mathrm{Cr}_{2-x} \mathrm{~S}_{4}(x=$ 0.4 ) are grown on glass substrate by chemical bath deposition technique. All AR grade chemicals from MERCK are used for growth of $\mathrm{Hg}_{x} \mathrm{Cr}_{2-x} \mathrm{~S}_{4}$ thin films. For the deposition of $\mathrm{Hg}_{x} \mathrm{Cr}_{2-x} \mathrm{~S}_{4}$, solutions of $\mathrm{HgCl}_{2}, \mathrm{CrO}_{3}$, and $\mathrm{NH}_{2}-\mathrm{CS}-\mathrm{NH}_{2}$ are prepared separately of concentration $0.1 \mathrm{M}$ using double distilled water as solvent and mixing them in stoichiometric proportion. EDTA is used as complexing agent. $\mathrm{pH}$ of the reaction mixture was adjusted by adding ammonia. The deposition was carried out in borosil glass beaker of capacity $100 \mathrm{~mL}$. It is used to put reactant in the form of solution; hence it is served as chemical reaction bath. This chemical reaction bath is put inside the constant temperature oil bath. The chemical reactant in the form of solution is stirred by 


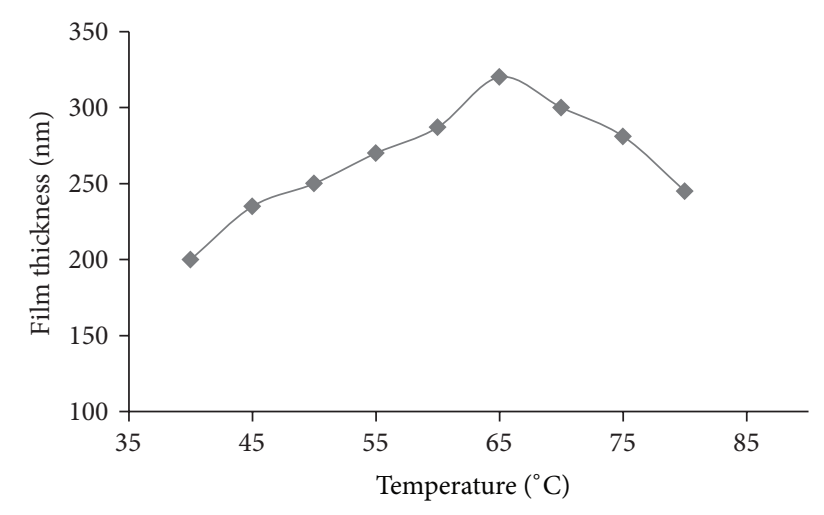

FIGURE 1: Optimization of solution bath temperature for $\mathrm{Hg}_{x} \mathrm{Cr}_{2-x} \mathrm{~S}_{4}(x=0.4)$.

magnetic stirrer. Well-cleaned glass microslides are dipped vertically in the chemical reaction bath by providing support to glass pot of the reaction bath. The stirring speed of magnetic stirrer is so adjusted that the solution can be stirred slowly during the deposition process. After deposition of $\mathrm{HgCr}_{2} \mathrm{~S}_{4}$ thin films, the substrates are taken out and washed with double distilled water and dried in air. Finally, they are preserved in an air-tight container. The thickness of deposited thin films is measured by the weight difference technique at room temperature. The structural properties of the films are analyzed by using Bruker AXS D8 Advanced model Xray diffractometer $\left(\mathrm{CuK}_{\alpha}\right.$ radiation; $\left.\lambda=0.15405 \mathrm{~nm}\right)$, and the grain size is determined from the Scherrer formula. The grown composition is analyzed by the EDAX technique.

\section{Results and Discussions}

\subsection{Impact of Preparative Parameters}

3.1.1. Impact of Bath Temperature. Figure 1 shows the variation of film thickness with deposition temperature, keeping other parameters constant. The temperature of chemical bath was changed from $40^{\circ} \mathrm{C}$ to $90^{\circ} \mathrm{C}$ with an interval of $5^{\circ} \mathrm{C}$. It can be seen from Figure 1 that the thickness goes on increasing with bath temperature; it reaches maximum thickness at $65^{\circ} \mathrm{C}$ and further decreases with increase in temperature after $65^{\circ} \mathrm{C}$ [12-14].

3.1.2. Impact of Complexing Agent. Figure 2 shows the variation of film thickness with complexing agent, keeping the other parameters same. The volume of EDTA was changed into a bath solution. The maximum and uniform thin films were obtained with the addition of $4.5 \mathrm{~mL}$ EDTA into a chemical bath solution and further decrease with increase in volume of EDTA.

3.1.3. Impact of Thiourea Concentration. Figure 3 shows the variation of film thickness with volume of thiourea, keeping other parameters the same. The volume of thiourea was changed into a bath solution. The maximum and welluniformed thin films were obtained with the addition of

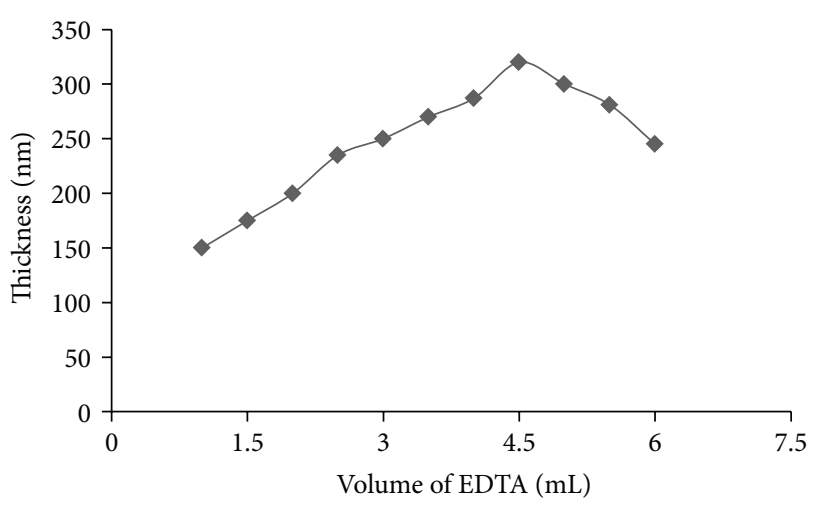

FIgURE 2: Optimization of volume of EDTA for $\mathrm{Hg}_{x} \mathrm{Cr}_{2-x} \mathrm{~S}_{4}(x=$ $0.4)$.

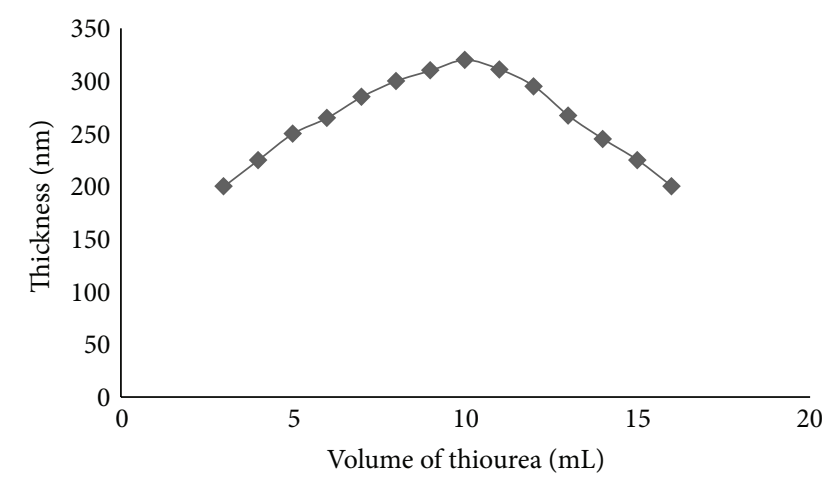

FIGURE 3: Optimization of volume of thiourea for $\mathrm{Hg}_{x} \mathrm{Cr}_{2-x} \mathrm{~S}_{4}(x=$ $0.4)$.

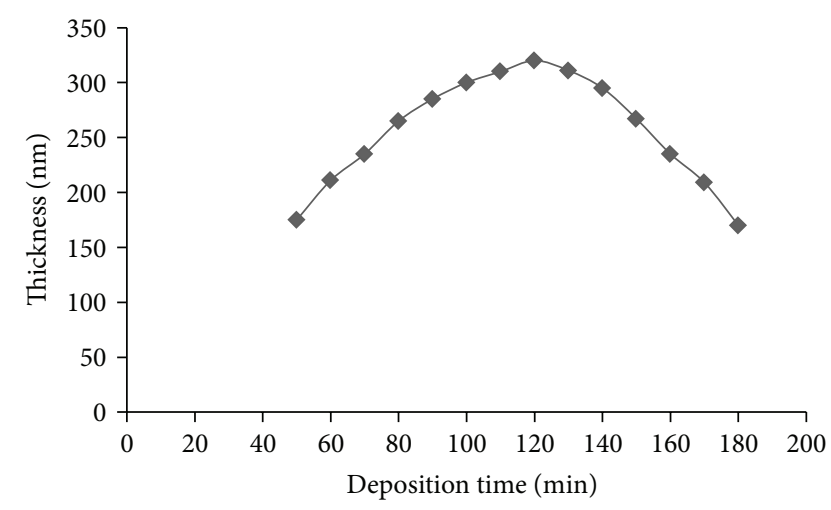

FIGURE 4: Optimization of deposition time for $\mathrm{Hg}_{x} \mathrm{Cr}_{2-x} \mathrm{~S}_{4}(x=$ $0.4)$.

$10 \mathrm{~mL}$ thiourea into a chemical bath solution. $\mathrm{pH}$ of the chemical bath solution was 10 at room temperature. Figure 3 indicates that the thickness went on increasing with volume of thiourea reaching to maximum $(10 \mathrm{~mL})$ and then decreases with further increase in volume of thiourea.

3.1.4. Impact of Deposition Time. The impact of deposition time on thickness was studied in Figure 4, keeping the other parameters the same. The thickness of thin film went on 


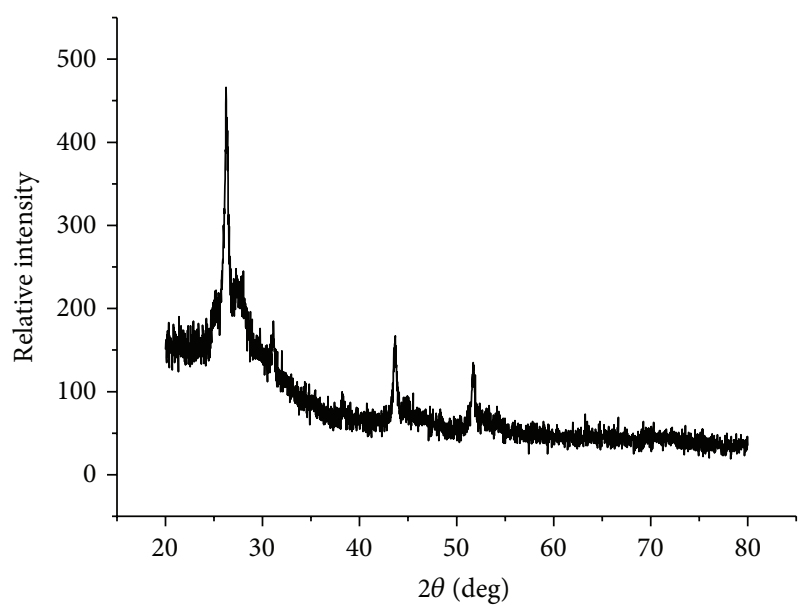

FIGURE 5: XRD pattern of $\mathrm{Hg}_{x} \mathrm{Cr}_{2-x} \mathrm{~S}_{4}(x=0.4)$ thin film.

TABLE 1: Experimental and standard " $2 \theta$ " and " $d$ " spacing.

\begin{tabular}{lcccc}
\hline $\begin{array}{l}\text { Planes } \\
(h k l)\end{array}$ & \multicolumn{2}{c}{ 2 Theta (degree) } & \multicolumn{2}{c}{$d$-spacing values $d(\AA)$} \\
\hline 220 & 24.572 & 24.580 & 3.62000 & 3.61967 \\
311 & 28.899 & 28.900 & 3.08700 & 3.08687 \\
331 & 38.269 & 38.260 & 2.35000 & 2.34875 \\
422 & 43.254 & 43.260 & 2.09000 & 2.08982 \\
\hline
\end{tabular}

TABLE 2: Elemental composition of $\mathrm{Hg}_{x} \mathrm{Cr}_{2-x} \mathrm{~S}_{4}(x=0.4)$ thin film deposited by chemical bath method.

\begin{tabular}{lcc}
\hline Element & Weight\% & Atomic\% \\
\hline $\mathrm{Hg}$ & 77.69 & 19.35 \\
$\mathrm{Cr}$ & 3.28 & 30.31 \\
$\mathrm{~S}$ & 19.03 & 50.34 \\
\hline
\end{tabular}

increasing with time of deposition, reaching to maximum at 120 minute.

\subsection{Structural Analysis}

3.2.1. X-Ray Diffractograph. XRD of the as grown films with optimum growth parameters was carried out. It is shown in Figure 5. The observed XRD pattern shows cubic crystal structure with noticeable growth along the (220) plane [15], in addition to the other small peaks, namely, (311), (331), and (422). Table 1 shows the observed and standard XRD data. This confirmed the formation of $\mathrm{Hg}_{x} \mathrm{Cr}_{2-x} \mathrm{~S}_{4}(x=0.4)$ [16].

3.2.2. EDAX Analysis. Table 2 shows the composition of element in $\mathrm{Hg}_{x} \mathrm{Cr}_{2-x} \mathrm{~S}_{4}$ thin films measured by EDAX analysis. The composition was found to be homogeneous and stoichiometric. Figure 6 shows EDAX spectrum of $\mathrm{Hg}_{x} \mathrm{Cr}_{2-x} \mathrm{~S}_{4}$ compound. The composition of element in thin films and in initial reactant in chemical bath is similar.

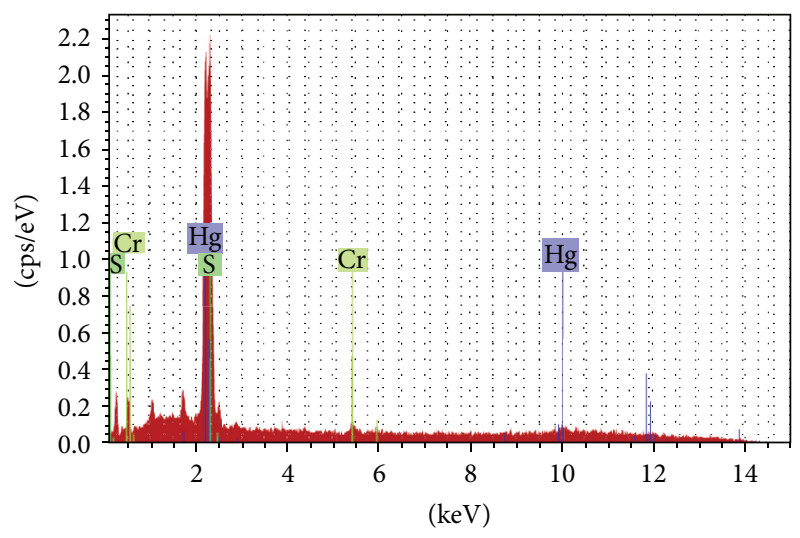

Figure 6: EDAX spectrum of a $\mathrm{Hg}_{x} \mathrm{Cr}_{2-x} \mathrm{~S}_{4}(x=0.4)$ compound.

\section{Conclusion}

In conclusion, $\mathrm{Hg}_{x} \mathrm{Cr}_{2-x} \mathrm{~S}_{4}(x=0.4)$ thin films can be deposited by simple chemical bath deposition technique. The films are sensitive to different growth parameters. The grown material is cubic crystal structure. The composition was found homogenous and stoichiometric. The optimum conditions are found to obtain $\mathrm{Hg}_{x} \mathrm{Cr}_{2-x} \mathrm{~S}_{4}$ films with maximum thickness.

\section{Acknowledgments}

The authors are thankful to Director, Professor Ajay Gupta, Dr. D. M. Phase, Dr. R. J. Chaudhari, Dr. N. P. Lalla, and Mukul Gupta, UGC-DAE Consortium for Scientific Research, Indore, for characterization work and valuable suggestions.

\section{References}

[1] M. A. Mahdi, S. J. Kasem, J. J. Hassen, A. A. Swadi, and S. K. J. A. I-Ani, "Structural and optical properties of chemical deposition CdS thin films," International Journal of Nanoelectronics and Materials, vol. 2, pp. 163-172, 2009.

[2] S. S. Kale and C. D. Lokhande, "Thickness-dependent properties of chemically deposited CdSe thin films," Materials Chemistry and Physics, vol. 62, no. 2, pp. 103-108, 2000.

[3] T. Kimura, T. Goto, H. Shintani, K. Ishizaka, T. Arima, and Y. Tokura, "Magnetic control of ferroelectric polarization," Nature, vol. 426, no. 6962, pp. 55-58, 2003.

[4] N. Hur, S. Park, P. A. Sharma, J. S. Ahn, S. Guha, and S.-W. Cheong, "Electric polarization reversal and memory in a multiferroic material induced by magnetic fields," Nature, vol. 429, no. 6990, pp. 392-395, 2004.

[5] T. Lottermoser, T. Lonkai, U. Amann, D. Hohlwein, J. Ihringer, and M. Fiebig, "Magnetic phase control by an electric field," Nature, vol. 430, no. 6999, pp. 541-544, 2004.

[6] T. Goto, T. Kimura, G. Lawes, A. P. Ramirez, and Y. Tokura, "Ferroelectricity and giant magnetocapacitance in perovskite rareearth manganites," Physical Review Letters, vol. 92, no. 25, pp. 257201-257204, 2004. 
[7] N. Hur, S. Park, P. A. Sharma, S. Guha, and S. -W. Cheong, "Colossal magnetodielectric effects in $\mathrm{DyMn}_{2} \mathrm{O}_{5}$," Physical Review Letters, vol. 93, no. 10, pp. 107207-107210, 2004.

[8] P. P. Sahay, R. K. Nath, and S. Tewari, "Optical properties of thermally evaporated CdS thin films," Crystal Research and Technology, vol. 42, no. 3, pp. 275-280, 2007.

[9] D. C. Cameron, W. Duncan, and W. M. Tsang, "The structural and electron transport properties of CdS grown by molecular beam epitaxy," Thin Solid Films, vol. 58, no. 1, pp. 61-66, 1979.

[10] S. M. Mahdavi, A. Irajizad, A. Azarian, and R. M. Tilaki, "Optical and structural properties of copper doped CdS thin films prepared by pulsed laser deposition," Scientia Iranica, vol. 15, no. 3, pp. 360-365, 2008.

[11] R. S. Mane, V. V. Todkar, C. D. Lokhande, J.-H. Ahn, and S.-H. Han, "Influence of strain on the surface wettability in crystalline $\mathrm{HgCr}_{2} \mathrm{~S}_{4}$ thin films," Nanotechnology, vol. 17, no. 21, article 018, pp. 5393-5396, 2006.

[12] S. H. Pawar and C. H. Bhosale, "Electrochemical bath deposition technique: deposition of CdS thin films," Bulletin of Materials Science, vol. 8, no. 3, pp. 419-422, 1986.

[13] V. Balasubramanian, N. Suriyanarayanan, and S. Prabahar, "Thickness-dependent structural properties of chemically deposited $\mathrm{Bi}_{2} \mathrm{~S}_{3}$ thin films," Advances in Apllied Science Research, vol. 3, no. 4, pp. 2369-2373, 2012.

[14] R. S. Mane, V. V. Todkar, C. D. Lokhande, S. S. Kale, and S.-H. Han, "Growth of crystalline $\mathrm{HgCr}_{2} \mathrm{~S}_{4}$ thin films at mild reaction conditions," Vacuum, vol. 80, no. 9, pp. 962-966, 2006.

[15] JCPD'S card no. 027-0316.

[16] V. V. Todkar, R. S. Mane, C. D. Lokhande, and S.-H. Han, "p-Type crystalline $\mathrm{HgCr}_{2} \mathrm{~S}_{4}$ semiconductor electrode synthesis and its photoelectrochemical studies," Journal of Photochemistry and Photobiology A, vol. 181, no. 1, pp. 33-36, 2006. 

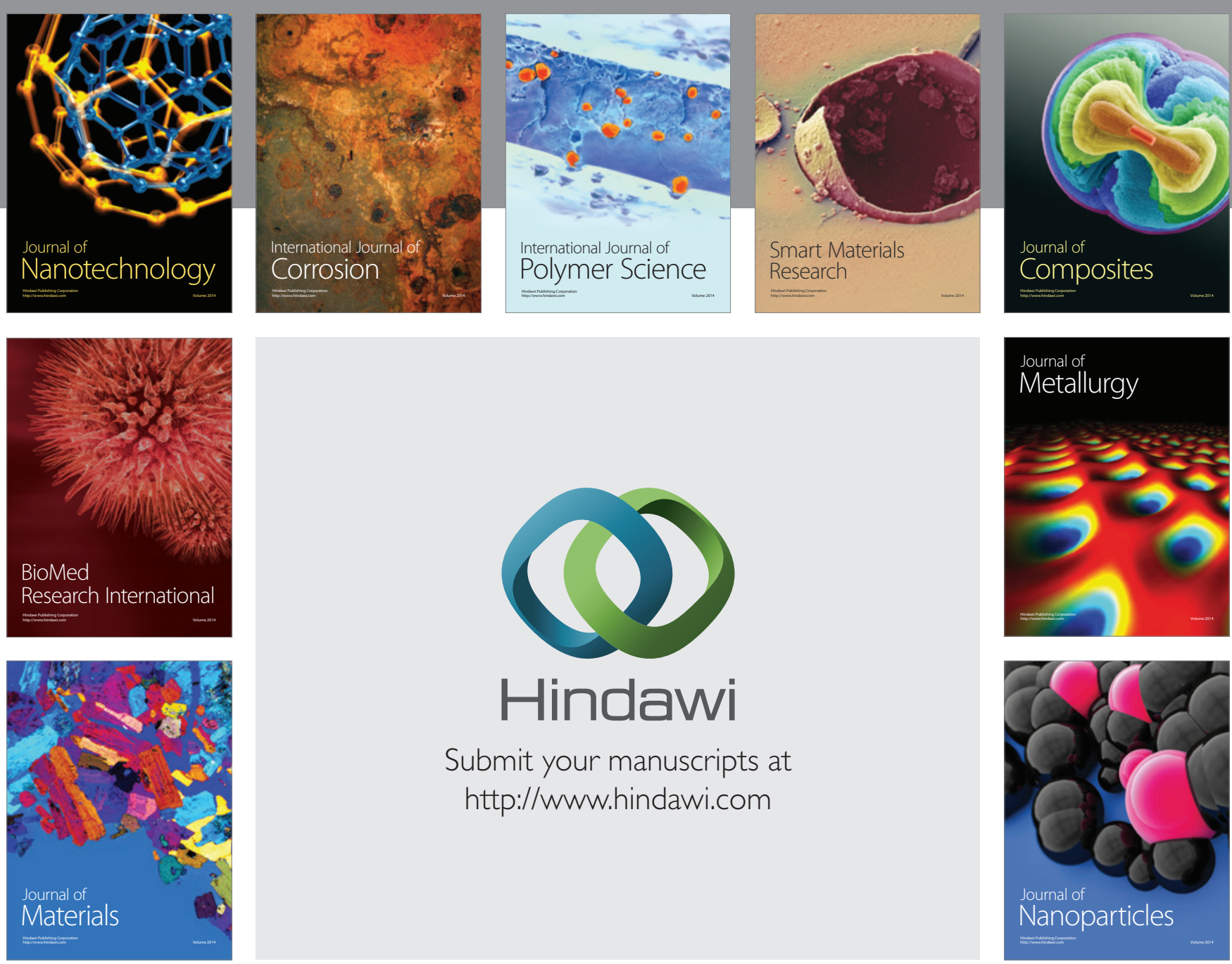

Submit your manuscripts at http://www.hindawi.com
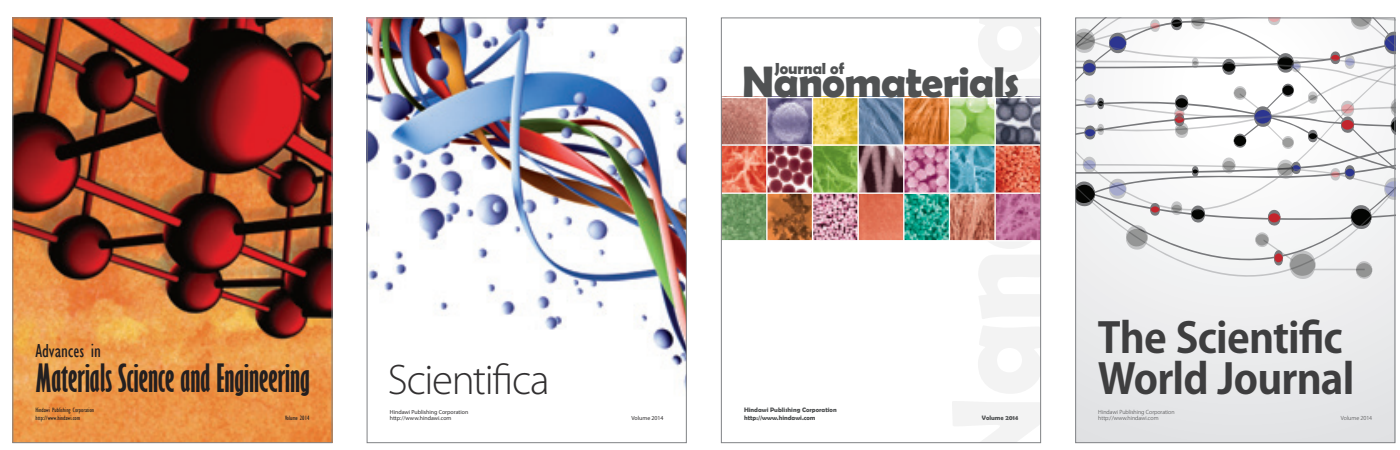

\section{The Scientific World Journal}
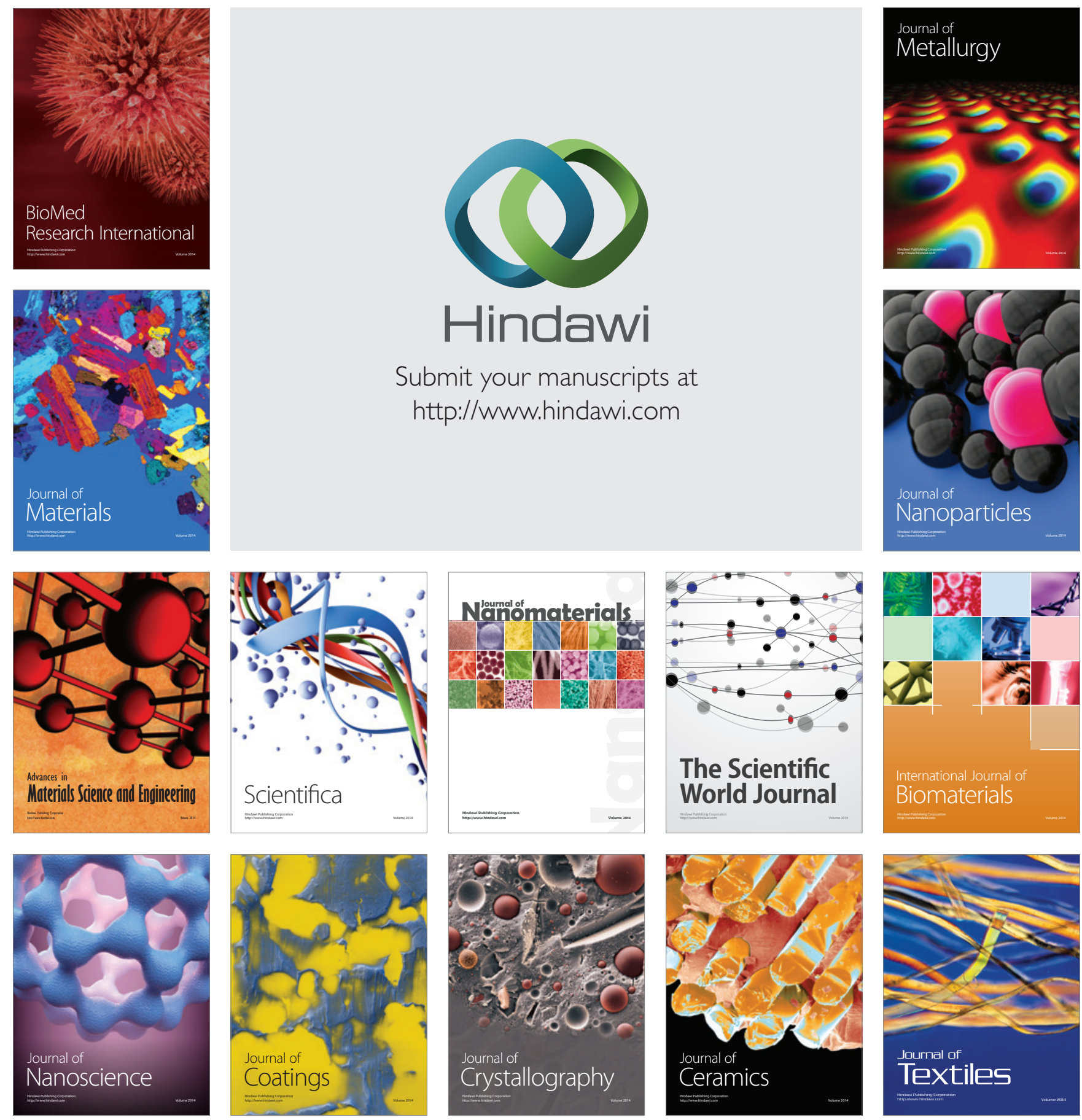